\title{
Ethical principles and standards for the conduct of biomedical research and publication
}

\author{
Kelvin K. L. Wong ${ }^{1,2} \cdot$ Steve C. N. Hui ${ }^{3}$
}

Received: 18 March 2015/Accepted: 18 March 2015/Published online: 13 August 2015

(C) Australasian College of Physical Scientists and Engineers in Medicine 2015

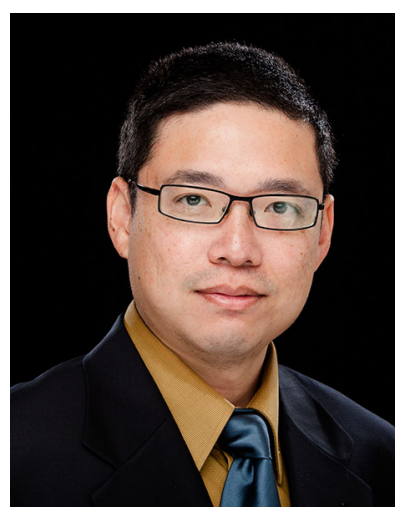

The importance and benefits of research ethics and guidelines

Clear and widely acceptable values and principles should be involved in scientific investigations. Scientific honesty is a fundamental aspect which leads to the overall quality of a study while misconduct involving cheating and plagiarism could cause controversy and could eventually lead to a

Kelvin K. L. Wong

kelvin.wong@uwa.edu.au; kwong@eleceng.adelaide.edu.au

1 Engineering Computational Biology, School of Computer Science and Software Engineering, The University of Western Australia, 35 Stirling Highway, Crawley, WA 6000, Australia

2 Centre for Biomedical Engineering, Department of Electronic and Electrical Engineering, University of Adelaide, Adelaide, SA 5005, Australia

3 Department of Imaging and Interventional Radiology, The Chinese University of Hong Kong, Sha Tin, N.T., Hong Kong, China tragedy. The aim of this letter is to promote principles and correct values to all scientists especially in research involving humans. In the field of biomedical engineering and medical physics, investigators, in many cases, initiate research related to living subjects and data. Protection of volunteers and their privacy becomes a primary and significant concern, and so conducting the research with good ethics inevitably becomes an important element for a successful study leading to valuable medical science publications.

New ideas, inventions, methods, discoveries and evidence are the core contributions of research to a society. Significant contributions of research are based on the quality and impact to the society. Carrying out a good research study with integrity helps to maintain the accuracy and reliability, which is managed by quality control and assurance. Quality assurance involves the establishment of standards, the execution of procedures, verification of data and improvement of any aspect in a research. The overall quality of a research could be improved if investigators follow the standard, commit to the protocol, monitor and audit all data and financial issues. One might not find an exact definition of research integrity, but when your research is conducted using high moral standard in common and core values and beliefs, strict principles and guidelines, with good quality control and assurance, investigators will be on the right track. This Letter will provide specific details on the principles of research integrity and good writing mannerism.

\section{The three pillars of good ethics in a research study involving human trials}

Subject protection, data integrity and scientific background and are the three major pillars of a good research study. Investigators should follow and obey guidelines, 
policies, regulations, local legal requirements, and ethics declarations. They have the responsibility to take care of all participants, protect their privacy and safety. Subject's participation is completely voluntary based on a completed informed consent and a signed agreement. All members of the research team should follow the protocol at all times. Data integrity is another major concern in clinical research. They should be consistent, accurate and reliable, and should not involve any non-scientific adjustment while doing analysis. Back to the fundamental of research, studies need to be carried out based on scientific evidence supported by proper hypotheses or pilot results which aim to eventually answer a question or solve a problem. With good ethical principles, investigators will conduct a proper research. Quality assurance and quality control also play a crucial role in research integrity, and investigators have the responsibility to ensure all aspects in a study and planned ahead in the protocol to manage and overview the whole project. With specification in the next section, we will detail the principles of good research conduct and integrity surrounding these three pillars.

\section{Principles of good research conduct and integrity}

Compliance to international research policies: Compliance with good research ethics and research practice is a top priority in publishing a scientific article. In the context of medical research, authors and investigators should be able to uphold the protection of research participants' rights and safety, and manage their data with integrity which refers to assuring the accuracy, consistency, and privacy of all data [1]. In certain types of studies especially when articles are involved, a third party as a contractor maybe hired to monitor the progress and data accuracy of drugs administration. Conflicts of interest should be declared especially when sponsors were involved among investigators and different parties to the research. Several well-known international research ethical guidelines including the Declaration of Helsinki, Belmont Report, Nuremberg Code and ICH-GCP provide comprehensive concepts and ideas of ethics to investigators [2-5]. Declaration of Helsinki involves 15 ethical categories such as risk management, subject's protection, informed consent, public registration and publication, scientific principles and research protocols, quality control etc. It has been well developed, modified and amended since 1960s and is regarded as one of the most complete ethical guidelines. A clinical research protocol should be in compliance with the Declaration of Helsinki. Belmont Report is developed based on the concepts of protection of human subjects regarding risks and benefits, respect and justice. Nuremberg code has a long history since the Second World War. Its principle is more focusing on the informed consent, voluntary participation, and the formulated scientific experimentation. ICH-GCP is a set of ethical standards for investigators and trial sponsors. Besides the protection of subjects and data, it states the role of investigators, sponsors and monitors, so researchers conducting drug trial studies should pay extra attention to this set of standards. Investigators may find it difficult to memorize all details in different ethical guidelines, so the following are some important concepts and ideas to be kept in mind.

\section{Ethical principles}

Ethical principles are developed based on the benefits to the society and the protections, well-being and willingness of the participants. All clinical research carries certain degrees of risk even a retrospective study which only involves patients' past records and data. To balance the risk-benefit ratio, investigators should evaluate the good for society, justify based on scientific knowledge and importance of the research topic. In protection of participants, foreseeable hazards, unnecessary suffering and injury should be avoided. Proper equipment, protection facility, and qualified persons to conduct the research should be well managed. Termination of research is recommended to avoid adverse events during the study and change of research protocol should be reported to the corresponding departments. All participants also have the rights to ask any questions regarding the research and to withdraw from the study without prejudice at any time [4].

\section{Informed consent from participants}

Participation in a research study is completely voluntary. The process of informed consent involves two parts: informing the potential participants about the research study with a complete and comprehensive explanation and details of the research; then followed by obtaining a signed consent form. Several essential elements must be contained in the Informed Consent Form including, but not limited to, (1) full title of the study, (2) full name and contact of the principle investigator, (3) name of the sponsor if applicable, (4) the objective, goals and purposes of the research, (5) details of any medical or clinical interventional involved, (6) arrangement and procedures, (7) data protection, (8) potential benefits and risks of the study, (9) the right and responsibility of participants, (10) cost or honorarium if applicable, (11) in layman terms and the preferred language to the participant sand (12) signature from the participants. [6] If any interventions involve radiation such as X-ray and $\mathrm{CT}$ scanning, potential risk and 
hazard have to be clearly stated in the consent form, and a medical physicist should be consulted in dosage measurement. Research involving the use of ionizing radiation should include a statement of compliance to the ARPANSA RPS8 which the protocol has to follow the recommendations and guideline. Informed consent is an agreement between participants and investigators which must be obtained before the start of any interventional or research activity.

\section{Research protocol}

Research protocol is a document that clearly defines the details, procedure and management of a research study. All investigators and members of the research team should follow the protocol while conducting the study. Changes to the protocol such as the change of study design, administration of articles, medical interventions, clinical interventional etc., are required to be reported to the corresponding ethics committee. A statement of compliance with widely accepted ethical declarations such as Declaration of Helsinki or the ICH-GCP should be included in the protocol.

\section{Permissions from regulatory agencies}

Principle investigators have the responsibility to seek approval from a regulatory agency, ethics committee or an institutional review board who review all documents including the informed consent form, research protocol, details of all investigators, declaration of conflict of interest, advertising materials, etc., prior conducting the research. To conduct a good clinical practice, Investigators should always keep a positive attitude and protect the wellbeing, welfare, rights and benefits to the participants. Investigators should also maintain a reasonable and high level of scientific background, and keep the integrity of research data.

Similar details have been reported in the National Statement on Ethical Conduct in Human Research (2007), which provides guidelines according to the National Health and Medical Research Council Act 1992. [7] Values, principles, risk, benefit, consent information should all been taken into account as part of the research study. A chapter of the statement specifically discusses about human specimens and investigators are suggested to overview it before the start of taking samples. Investigators should also pay extra attention when recruiting volunteers involving special groups such as under-aged youth and children, patients with mental disability and people who are highly dependent on medical care, since they are unable to give their own consent.

\section{Principles of honest writing conduct}

Following the completion of your research, the next step is to translate your hypothesis, methodologies, findings, and conclusion into an acceptable style of presentation, and with integrity and honesty. To conduct research in science and technology with good ethics and to build good academic atmosphere of academic periodicals, one should submit high quality original works that have not been considered, accepted or published in other journals or conference proceedings including text, data, figures, tables, illustrations and any copyright materials.

Ideas, methods, figures, findings, or results from other publications used in your work must be cited properly in an appropriate position, or it could be considered as plagiarism. Any articles with evidence of plagiarism or deceit, should be rejected with written warning. In serious cases, authors could be punished by permanent refusing and their corresponding institutions should be informed. Academic misconduct is indicated in authors' track records which strongly affect the reputation not only to the investigators but also to their department and institution. Prequalifying check will be conducted if a research group or department has a history of frequent academic misconduct. A redundant or duplicated manuscript will be rejected immediately as it violates the policies of journal.

All authors of a manuscript should have a substantial contribution to the research study and be responsible for the submitted manuscript. It is the authors responsibility to ensure all content in a manuscript, data and results are accurate and reliable, and the absence of academic misconduct. Conflicts of interest, sponsors, and any other kinds of financial supports need to be disclosed when the manuscript is published.

If authors discover mistakes or information that should be disclosed, they have the responsibility and obligation to timely send in editorial letters on the errors, make the amendment or even request for withdrawal. The duty of strengthening the academic moral construction and research integrity, creating a good academic atmosphere, as well as promoting the development of science and technology and social progress together lie with all of us.

\section{Summary}

Scientific research is evidence-based and encourages new concepts and ideas, and should have no tolerance of academic cheating and plagiarism. To be a good investigator, one should be responsible for all aspects within the research and at the study site and adhere to international policies and ethical principles. We have highlighted clearly 
the principles of good research conduct and integrity research morale leading to healthy publications in the biomedical engineering and science domains. It presents a very important set of principles for benchmarking your research articles against an internationally acceptable standard.

\section{References}

1. Clinical Effectiveness and Technology Management Department QaSD, editor Clinical Research Ethics and Good Clinical Practice and Clinical Research Legal and Regulatory Affairs. 2015 HA Training Symposia on Clinical Research Compliance; 2015 1/29/ 2015 and 2/11/2015; Hospital Authority Head Office, Hospital Authority Head Quarter, Hong Kong

2. Association WM (2013) WMA declaration of Helsinki: ethical principles for medical research involving human subjects. http:// www.wma.net/en/30publications/10policies/b3/index.html. Accessed 26 March 2015

3. Protection of human subjects; Belmont Report: notice of report for public comment (1979) Federal register 44(76):23191-7

4. The Nuremberg Code, United States Department of Health and Human Services (2005). http://www.hhs.gov/ohrp/archive/nur code.html. Accessed 26 March 2015

5. The International Conference on Harmonisation of Technical Requirements for Registration of Pharmaceuticals for Human Use (1996) Good Clinical Practice. http://www.ich.org/products/guide lines/efficacy/efficacy-single/article/good-clinical-practice.html. Accessed 26 March 2015

6. The Joint Chinese University of Hong Kong (2010) New Territories East Cluster Clinical Research Ethics Committee . http://www.crec. cuhk.edu.hk/. Accessed 26 March 2015

7. Australian Government NHaMRC (2007) National Statement on Ethical Conduct in Human Research. https://www.nhmrc.gov.au/ guidelines-publications/e72. Accessed 26 March 2015 